\title{
On the secondary charging effects and structure of mesospheric dust particles impacting on rocket probes
}

\author{
O. Havnes and L. I. Næsheim \\ Institute of Physics, University of Troms $\varnothing$, Norway \\ Received: 26 May 2006 - Revised: 12 February 2007 - Accepted: 6 March 2007 - Published: 29 March 2007
}

\begin{abstract}
The dust probe DUSTY, first launched during the summer of 1994 (flights ECT-02 and ECT-07) from Andøya Rocket Range, northern Norway, was the first probe to unambiguously detect heavy charged mesospheric aerosols, from hereon referred to as dust. In ECT-02 the probe detected negatively charged dust particles in the height interval of 83 to $88.5 \mathrm{~km}$. In this flight, the lower grid in the detector (Grid 2) measures both positive and negative currents in various regions, and we find that the relationship between the current measurements of Grid 2 and the bottom plate can only be explained by influence from secondary charge production on Grid 2. In ECT-07, which had a large coning, positive currents reaching the top grid of the probe were interpreted as due to the impact of positively charged dust particles. We have now reanalyzed the data from ECT- 07 and arrived at the conclusion that the measured positive currents to this grid must have been mainly due to secondary charging effects from the impacting dust particles. The grid consists of a set of parallel wires crossed with an identical set of wires on top of it, and we find that if the observed currents were created from the direct impact of charged dust particles, then they should be very weakly modulated at four times the rocket spin rate $\omega_{R}$. Observations show, however, that the observed currents are strongly modulated at $2 \omega_{R}$. We cannot reproduce the observed large modulations of the impact currents in the dust layer if the currents are due only to the transfer of the charges on the impacted dust particles. Based on the results of recent ice cluster impact secondary charging experiments by Tomsic (2003), which found that a small fraction of the ice clusters, when impacting with nearly grazing incidence, carried away one negative charge $-1 e$, we have arrived at the conclusion that similar, but significantly more effective, charging effects must be predominantly responsible for the positive currents measured by the top grid in ECT-07 and their large rotational modulation at $2 \omega_{R}$.
\end{abstract}

Correspondence to: O. Havnes

(ove.havnes@phys.uit.no)
Since the secondary effect is dependent on the size of the impacting dust, this opens up for the possibility of mapping the relative dust sizes throughout a dust layer by comparing the observed direct and secondary currents.

Keywords. Atmospheric composition and structure (Aerosols and particles; Instruments and techniques)

\section{Introduction}

Probes on rocket payloads have been essential in obtaining information about the conditions in much of the mesosphere. This region, between $\sim 50 \mathrm{~km}$ and $100 \mathrm{~km}$, cannot be reached by planes, balloons, or satellites, and in-situ measurements can only be done by rocket payloads. In addition to obtaining in-situ measurements, their high spatial resolution, which can be a few times $10 \mathrm{~cm}$, is also of vital importance in understanding the various processes which occur in these regions.

During the last few decades, it has become increasingly clear that dust particles, most likely consisting mainly of water ice, play a decisive role for some mesospheric phenomena, such as the noctilucent clouds (NLC) and its associated radar phenomenon Polar Mesospheric Echoes (PMSE) (e.g. Gadsden and Schröder, 1989; Cho and Röttger, 1997; Rapp and Lübken, 2004). The electron bite-outs, which are prominent large-scale reductions of electron density, were early suspected to be due to absorption of electrons by small dust particles (Pedersen et al., 1969; Ulwick et al., 1988). Direct observations of charged dust by a new type of dust probe (DUSTY) revealed that during PMSE conditions, where no visual dust was observed, large amounts of sub-visual dust were still present (Havnes et al., 1996). Later, rocket probe and lidar observations confirmed that charged dust is probably always present during PMSE conditions. They can apparently be both negatively and positively electrically charged (Havnes et al., 1996; Mitchell et al., 2001; Smiley et al., 2006). The dust radius is found to be from $\sim 70-80 \mathrm{~nm}$ and

Published by Copernicus GmbH on behalf of the European Geosciences Union. 


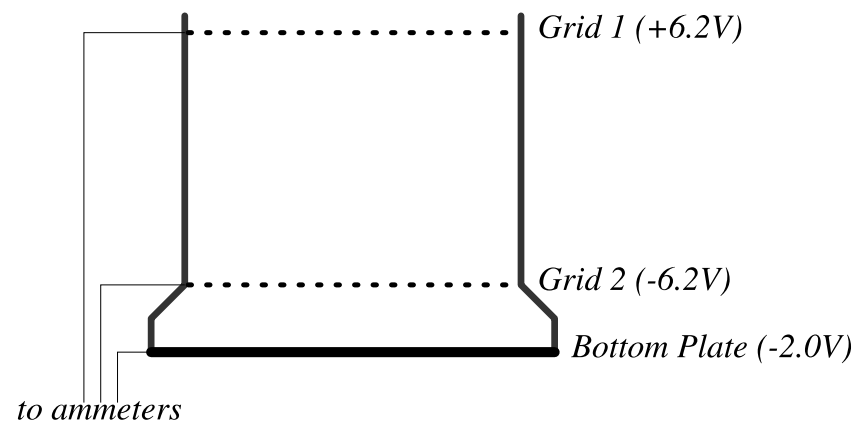

Fig. 1. The principle of the DUSTY probe, with its grid 1 to close the interior for ambient plasma, grid 2 to detect the effect of secondary charge production, and the bottom plate which will mainly detect directly impacting charged dust particles.

downwards (von Cossart et al., 1999). Theoretical work, based on the fact that 50-100 tons of meteoritic material burns out each day as meteors and enters the Earth's atmosphere with large velocities, predicts that ablation and recondensation of meteoritic material into "smoke particles" may produce a more or less persistent background of small and numerous dust particles (Hunten et al., 1980).

The presence of dust particles allows for the possibility that impacts of such particles on rocket payloads and its probes can lead to secondary charging effects which can dominate the observed signal and lead to misinterpretation of the real physical conditions.

It appears likely that several rocket measurements have been severely influenced by secondary effects due to impacts (Zadorozhny et al., 1993; Vostrikov et al., 1997; Gumbel and Witt, 1998). Because the relative velocity between the rocket and the atmospheric dust normally is modest, of the order of $1 \mathrm{~km} / \mathrm{s}$ or less, a direct electron secondary emission is not important. This requires impact velocities generally of the order of $10 \mathrm{~km} / \mathrm{s}$ or higher (e.g. Dalmann et al., 1977). At impact velocities of a few $\mathrm{km} / \mathrm{s}$ or less the most important secondary charging effects probably occur when dust particles strike a surface at a comparatively large incident angle, with the normal to the surface, and that a fraction of the particles leaving the surface carries away ions or electrons from the surface (e.g. Gridin et al., 2004; Andersson and Pettersson, 1997). Tomsic (2003) investigated the impact of cluster beams consisting of ice particles of radii up to $14 \mathrm{~nm}$ on various surfaces. When the ice particles impacted with low impact angles (i.e. nearly perpendicular to the surface), they tended to sublimate completely. At high impact angles, $\theta_{i} \gtrsim 45^{\circ}$, the particles larger than 6-7 nm sublimate only partially and large fragments may survive and leave the surface. Tomsic found that a small fraction of these surviving fragments can be charged during the impact. Particles smaller than 6-7 nm tend to evaporate completely. On fresh surfaces that have not been exposed to particle impacts, typically one in $10^{3}$ of the surviving fragments is negatively charged. After an exposure of some minutes, the emission of positively charged fragments dominates. Although it appears most likely that the dust in NLC and PMSE consists mostly of water ice, they must be much more effective than the pure water ice clusters in producing secondary charging, if they are to explain the positive currents on the dust probe grids as observed by Havnes et al. (1996) in the two rocket flights ECT-02 and ECT-07.

In the following, we will re-examine the measurements of dust impacts made in these two flights. We will first consider ECT-02. In this flight the payload had little coning and the DUSTY probe worked as intended, detecting the presence of negatively charged subvisual dust and also showing that electron bite-outs were coinciding with particularly high dust charge density. We will examine if the positive currents observed by G2 in some height intervals require a significant secondary production or whether the simultaneous presence of both negative and positive dust particles at such heights can explain the observations. We will thereafter consider ECT-07, which had such a large coning $\left(\geq 42^{\circ}\right)$ that the DUSTY probe did not function as intended, since the interior of the probe was hidden for the incoming dust particles by the probe walls. The positive current measured on grid G1 (Grid 1 in Fig. 1), whose normal function is to close the dust probe to the ambient ion-electron plasma, was originally interpreted as due to the impact of positively charged dust particles (Havnes et al., 1996). This conclusion was apparently supported by electron probe measurements on the same payload showing an increase in the electron current as the payload passed the layer which gave the positive current to G1. We now reinterpret these measurements with a detailed modelling of the impact on the grid surfaces and the motion of their ejected fragments, all as a function of the payload rotation angle, where we compare the observed strongly rotationally modulated positive current to G1 with models with and without secondary charge production.

\section{A new look at the ECT-02 measurements}

The schematics of the dust probe are shown in Fig. 1. The probe is constructed to work at low coning angles so that dust particles will impact the bottom plate. The top grid G1 is at $+6.2 \mathrm{~V}$ and will close the probe to outside thermal ions or electrons by deflecting the positive ions and absorbing the electrons. The lower grid $\mathrm{G} 2$ is at $-6.2 \mathrm{~V}$ and was originally meant to detect possible secondary charges if produced by the dust impact on the bottom plate. If secondary production did happen, the measurements from G2 could be used as described by Havnes et al. (1996) for a correction, and thereby give the true current caused by the charged dust impact on the bottom plate. This current will then directly give the ambient dust charge density when the rocket velocity and probe dimensions are known. However, secondary production by the dust impact on the bottom plate at low impact angles $\theta_{i}$ 

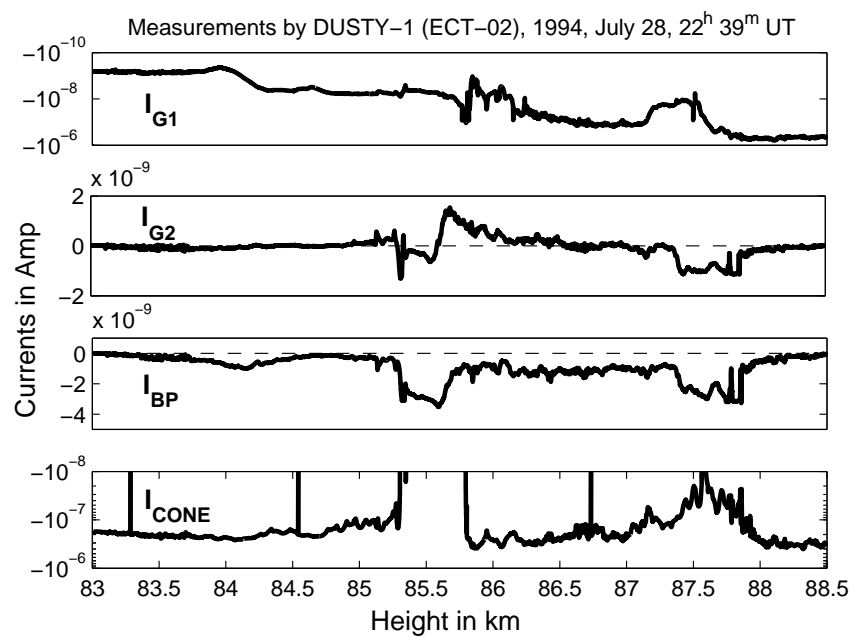

Fig. 2. Four currents measured by the rocket flight ECT-02 on upleg. From top panel and down: Current to grid 1, grid 2 and the bottom plate in the DUSTY probe in front of the payload. The electron current measured by the CONE instrument, at the rear of the payload.

to the normal of the bottom plate should not produce secondary charges at the low impact velocities of the order of $1 \mathrm{~km} / \mathrm{s}$ or less. We find that the currents measured by G2 must be a combination of the direct impacts from incoming dust, and of secondary production due to the incoming dust striking the cylindrical grid wires of G2 at high $\theta_{i}$.

In Fig. 2 we show the observed currents $I_{\mathrm{G} 1}, I_{\mathrm{G} 2}$ and $I_{\mathrm{BP}}$ to grids $\mathrm{G} 1, \mathrm{G} 2$, and to the bottom plate and $I_{\mathrm{CONE}}$ to the CONE electron probe (Blix et al., 1990). In Fig. 3 we show the ratio $R_{S}=I_{\mathrm{G} 2} / I_{\mathrm{BP}}$.

We will examine the observations in Figs. 2 and 3 on the basis of two different models for the dust population. Model 1 assumes that the currents are formed from direct impacts of dust particles which can be both positively and negatively charged, and that secondary effects are negligible. Model 2, on the other hand, assumes that the incoming dust particles are negatively charged but that secondary charges can influence the observed currents, which can lead to positive currents on the grids.

According to model 1 , the ratio $I_{\mathrm{G} 2} / I_{\mathrm{BP}}$ is formed by direct impacts and is

$R_{S}=\frac{I_{\mathrm{G} 2}}{I_{\mathrm{BP}}}=\frac{-\sigma I_{-}+\alpha I_{+}}{-(1-\sigma) I_{-}+(1-\alpha) I_{+}}$.

The currents $I_{-}$and $I_{+}$are the absolute value of the currents due to negative and positive dust in the region in front of G2, i.e. which has passed through G1. The fraction of the probe opening covered by the grid is $\sigma=0.23$. We have introduced another parameter $0.23 \leq \alpha \leq 1$ for the positive current to allow for the possibility that the positively charged dust particles are small enough, and have low enough kinetic energy, so that they can be attracted efficiently to the neg-

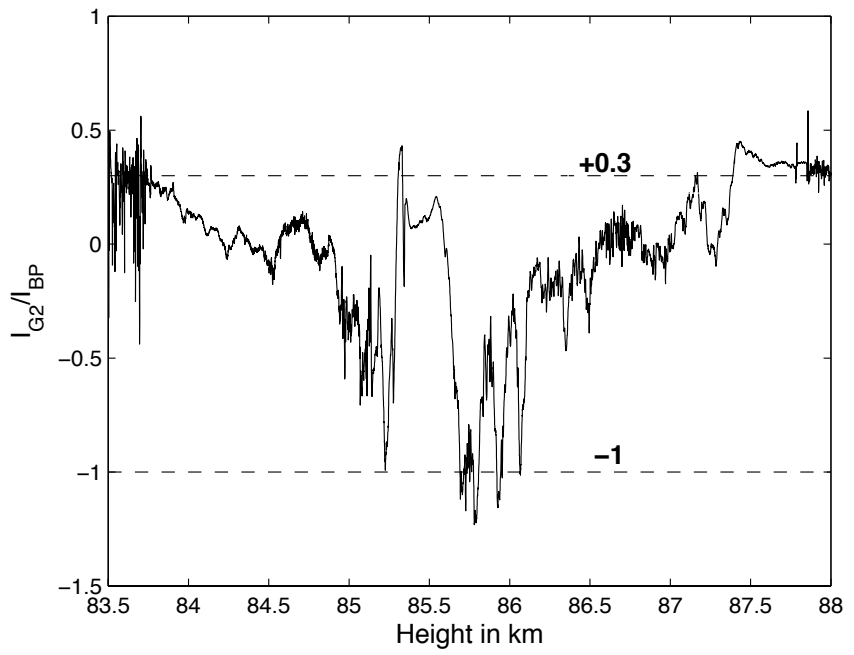

Fig. 3. The figure shows the ratio between the current to grid 2 and the bottom plate. The limit 0.3 is from Eq. (4) when secondary production is negligible while -1 is when it is dominant.

ative grid 2. If their energy is high, then $\alpha=\sigma=0.23$, and the ratio $R_{S}=\frac{\sigma}{1-\sigma}=0.3$. This does not fit well with the results of Fig. 3, except at the low and high points in the layer. We have assumed that the majority of the negatively charged dust is energetic enough to hardly be affected by the probe potentials. For the values of $R_{S}$ down to $\sim-1.2$, which are also observed, the only way this can be produced by model 1 is for $\alpha>0.23$, so that a larger fraction of the positive dust particles is attracted to and hits G2, while a corresponding smaller fraction reaches the bottom plate. To stay within the limits $R \sim 0.3$ to $R \sim-1$, as observed, requires that $I_{+} \leq I_{-}$. It is certainly possible, but not very likely, that $I_{+}$should be restricted only to values from $I_{-}$and smaller, and also that all such values are evidently found somewhere in the PMSE layer.

In the upper part of the bite-out we see that grid 2 measures its maximum positive current while $I_{\mathrm{BP}}$ is negative, as it is in the whole dust layer. The CONE electron probe current in the bite-out is below the detection limit, showing that the electron density is very low (Blix, 1999). For the model to explain the ratio $R_{S} \sim-0.8$, it is required that $I_{+}$is comparable to $I_{-}$, and that the positive dust is attracted to G2, so that $\alpha>\sigma$. In view of the near absence of free electrons, charge neutrality requires that

$N_{d-} Z_{d-}+N_{d+} Z_{d+}+n_{i+}-n_{i_{-}} \approx 0$

when we include positive and negative dust of density $N_{d-}$ and $N_{d+}$, respectively, with charge numbers $Z_{d-}$ and $Z_{d+}$. The positive and negative ion density is $n_{i+}$ and $n_{i-}$. If we neglect recombination with electrons since they have a very low density, the positive ion density $n_{i+}$ will now be given 
by

$q=\alpha_{-} n_{i+} n_{i-}+\pi r_{d}^{2} v_{i+} N_{d-} n_{i+}\left(1-\frac{e U_{-}}{k T_{i}}\right)$.

We use a pair production of $q=6 \times 10^{7} \mathrm{~m}^{-3} \mathrm{~s}^{-1}$ and a recombination rate for positive to negative ions of $\alpha_{-}=6 \times 10^{-14} \mathrm{~m}^{3} \mathrm{~s}^{-1}$ (Brasseur and Solomon, 1986). The ion thermal velocity $v_{i+} \sim 250 \mathrm{~m} / \mathrm{s}$ and the focusing effect of the negative dust on the positive ions is $\left(1-\left(e U_{-} / k T_{i}\right)\right) \sim 10$, meaning that the effective cross section of the dust for the capture of positive ions is $\sim 10$ times its geometric cross section. Under normal conditions with a dust radius of $r_{n m}=10 \mathrm{~nm}$ and $N_{d-} \sim 10^{9} \mathrm{~m}^{-3}$, with $n_{i-} \sim 10^{9} \mathrm{~m}^{-3}$, the capture of ions by dust will dominate. Considering the dust term only, we obtain $n_{i+} \sim 10^{10} \mathrm{~m}^{-3}$, while if we use recombination with negative ions only, we obtain $n_{i+} \gtrsim 2 \times 10^{11} \mathrm{~m}^{-3}$, if $n_{i-}<10^{9} \mathrm{~m}^{-3}$. This indicates that the positive ion density by model 1 must be high, also when compared to the electron density $n_{e 0}$ that we would have had if dust was not present. We would obtain $n_{e 0} \sim(q / \alpha)^{1 / 2} \sim 4 \times 10^{9} \mathrm{~m}^{-3}$ if for the positive ion-electron recombination rate we used $\alpha=4 \times 10^{-12} \mathrm{~m}^{3} \mathrm{~s}^{-1}$ (Brasseur and Solomon, 1986). If we use $n_{i+} \sim 10^{10} \mathrm{~m}^{-3} \gg n_{i-}$, we have from Eq. (2) that $N_{d-}\left|Z_{d-}\right| \sim N_{d+} Z_{d+}+n_{i+}$ which requires, for any reasonable choice of $N_{d-}\left|Z_{d-}\right|$ that $N_{d_{-}}\left|Z_{d_{-}}\right| \gg N_{d+} Z_{d+}$. Such densities cannot produce values like the observed $R_{S} \approx-0.8$ in the higher part of the bite-out, but would lead to $R \approx 0.3$. This means that it will strongly violate the requirement for plasma neutrality, if we attempt to describe the simultaneous positive current to $\mathrm{G} 2$ and negative current to the bottom plate by co-existing positive and negative dust particles of roughly the same charge density when, at the same time, the free electron density is very low.

Model 2, with production of secondary charges for the dust particles hitting G2 with a reasonably high impact angle, will directly explain the two situations we have discussed, as well as Fig. 3, where model 1 seems not to be the answer.

For model 2 the ratio is

$R_{S}=\frac{I_{\mathrm{G} 2}}{I_{\mathrm{BP}}}=\frac{-\sigma I_{-}+I_{S}}{-(1-\sigma) I_{-}-I_{S}}$.

Here $I_{S}$ is the absolute value of the secondary current produced by negative dust impact on G2. We do not include any positive dust. If the ratio $I_{S} / I_{-}$is zero (no secondary production), then we obtain the result $R_{S}=0.3$. Since secondary production decreases with decreasing dust size, this situation will be found if the dust particles are small. Such situations are found at the upper and lower edges of the dust layer and also within the bite-out. If the dust particles are large, and if the secondary current can be much higher than the direct current, so that $I_{S} / I_{-} \gg 1$, we obtain $R_{S} \rightarrow-1$. This shows that the observed ratio $R_{S}$ of Fig. 3 is within values which are close to the theoretical limits of model 2 (Eq. 4), which include secondary charge production, and that this is naturally explained as a variation of dust size within the dust layer. The relative dust sizes in the layer, as indicated by our results are physically plausible, with the smallest dust high up in the layer (where we believe they start to form), low (where they are gradually sublimating as they fall, due to an increased temperature) and also within the lower part of the electron bite-out (where approximately all electrons are locked on to grains, a situation which is most easily achieved if there are many small dust particles).

The situation where we measure a positive $I_{\mathrm{G} 2}$, negative $I_{\mathrm{BP}}$ and a very small $n_{e}$ is also directly explained by model 2 without violating the requirement of charge neutrality. In the bite-out, most of the electrons are captured by dust, and a majority of the dust particles are therefore most likely small. If the dust particles are large, then they must have an unrealistically high density to be able to absorb a sufficiently large fraction of the electrons. In the lower half of the bite-out our model predicts that few large dust particles are present, but in the upper part we need a non-negligible density of larger particles to also produce secondary effects on grid 2. We now compute, as described in the Appendix, the production of secondary charging by an effective grid area, which when multiplied by the influx of primary dust particles gives the number of negative fragments produced per second. For all 206 squares of grid 2, the total effective area is well represented by

$A_{\text {tot }}=5 \times 10^{-4} \eta_{S}(\max ) \mathrm{m}^{2}$.

In Eq. (5) we used for the secondary production curve the angles [35, 60, 85] (Fig. 6), and a payload coning angle of $\gamma=20^{\circ}$. The value $\eta_{S}(\max )$ on the production curve (which gives the number of fragments of an incoming dust particle which carry away an electron after impact) depends on the size of the incoming dust particles, and we will assume that the large dust particles dominate the production of secondary charging. Since the ratio between $A_{\text {tot }}$ and the total area of grid 2 is $A_{\text {tot }} / \sigma \pi R_{p r}^{2}=0.28 \eta_{S}$, and we now require that the secondary production by the large dust be larger than the direct current by the small and large dust combined, we must have

$$
\frac{I_{S}}{\sigma I_{-}} \sim \frac{0.28 \eta_{S}(\max ) N_{d L}}{N_{d L}\left|Z_{L}\right|+N_{d S}\left|Z_{S}\right|}>1 .
$$

Here the number density of large and small dust particles are $N_{d L}$ and $N_{d S}$, respectively, while their charge numbers are $Z_{L}$ and $Z_{S}$. Using $\eta_{S}(\max ) \sim 100$ we find that Eq. (6) requires that $N_{d L}>0.04 N_{d S}$ if we also use $\left|Z_{L}\right|=3$ and $\left|Z_{S}\right| \approx 1$. If the maximum small dust number density is $N_{d S} \simeq 4 \times 10^{9} \mathrm{~m}^{-3}$ (Havnes et al., 1996), then the corresponding large dust density is $N_{d L}>1.6 \times 10^{8} \mathrm{~m}^{-3}$. This shows that both the lack of electrons and the positive current measured on $\mathrm{G} 2$ can be explained directly if the small dust number density is high, but that there is also a $\gtrsim 10 \%$ density of large dust particles. Model 2 can therefore explain the observations, 

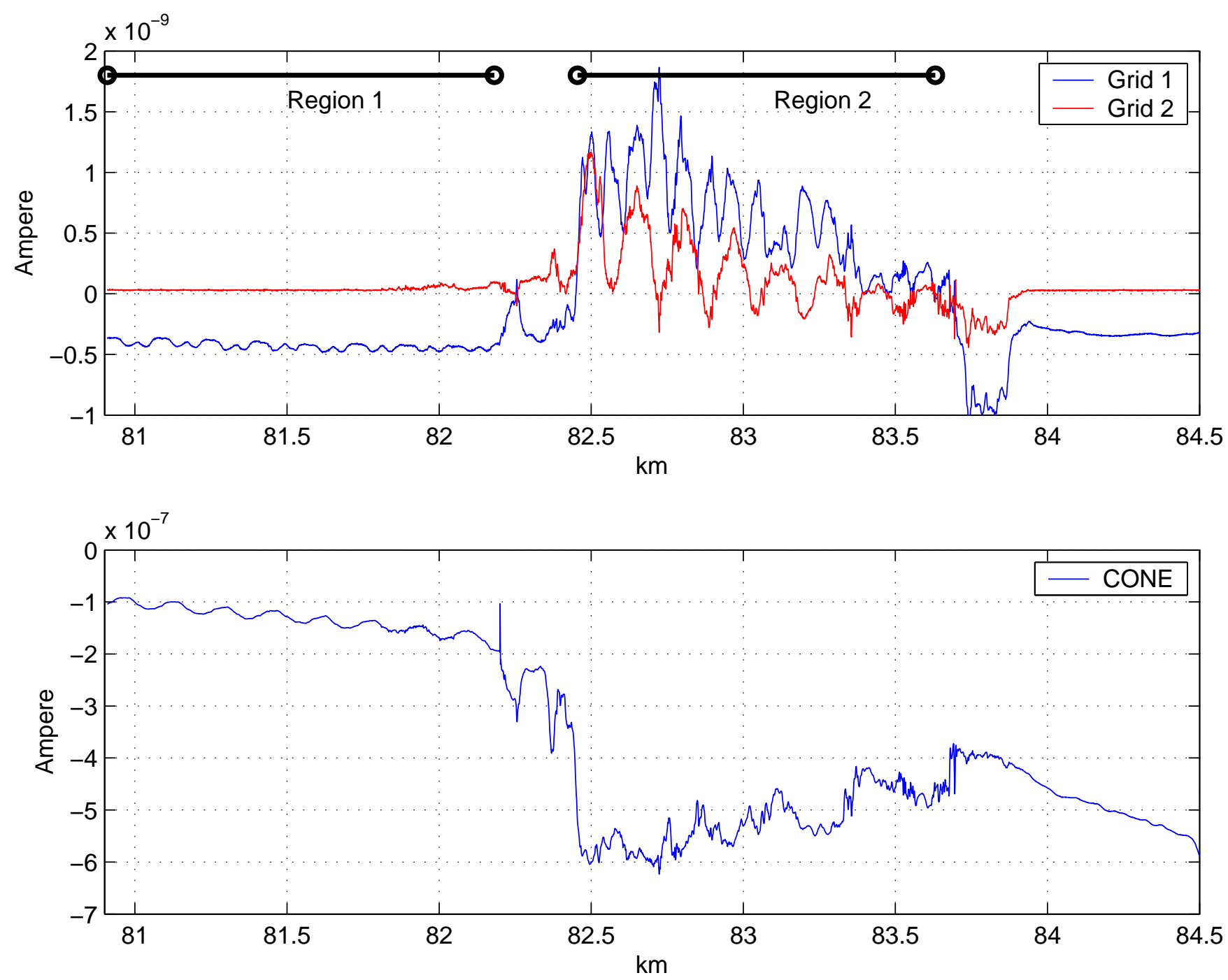

Fig. 4. The currents to grid 1 and grid 2, as measured by the DUSTY probe, and the electron current measured by the CONE probe, all on the flight ECT-07 (DUSTY 2).

provided that the production factor $\eta_{S}$ has a maximum value in the range $50-100$.

\section{Analysis of the currents to the front grid of DUSTY2 (ECT-07)}

On the flight ECT-07, the high coning led to most of G2 and the bottom plate being screened by the probe walls, so only G1 received the direct impact of dust particles. The original assumption, used when the measurements of ECT-07 were first analyzed (Havnes et al., 1996), was that the current $I_{\mathrm{G} 1}$ was due to the direct impact of positively charged dust particles transferring their net charge to G1. Since the potential of $\mathrm{G} 1$ is $+6.2 \mathrm{~V}$, the thermal positive ions, with an average kinetic energy of $\sim 0.01 \mathrm{eV}$, could not contribute to the positive current. At the comparatively low heights of this PMSE, the electrons contribute a modest negative current, as can be seen from Fig. 4 for the currents to G1 in region 1, outside the dust layer. If the measured positive current was due to the direct impact of particles, then this could only be due to impacting positively charged dust particles. If we assume a density of the dust material of $\rho_{d}=1000 \mathrm{~kg} \mathrm{~m}^{-3}$, all dust particles of radius $r_{d}>0.8 \mathrm{~nm}$ would, at the rocket velocity of $V_{R} \sim 900 \mathrm{~m} / \mathrm{s}$, overcome the G1 potential if the dust particles had a charge of $+1 e$. If the charge was, for example, $+100 e$ the size would have to be $r_{d}>4 \mathrm{~nm}$ in order to impact G1. However, one factor which was not given enough attention in the early analysis was the strong modulation of the G1 signal by the rotation of the payload (Fig. 4). A frequency analysis (FFT) of the observations, shown in Fig. 5, demonstrates that the frequency power spectrum is different below the dust layer compared to what it is inside the dust layer. 

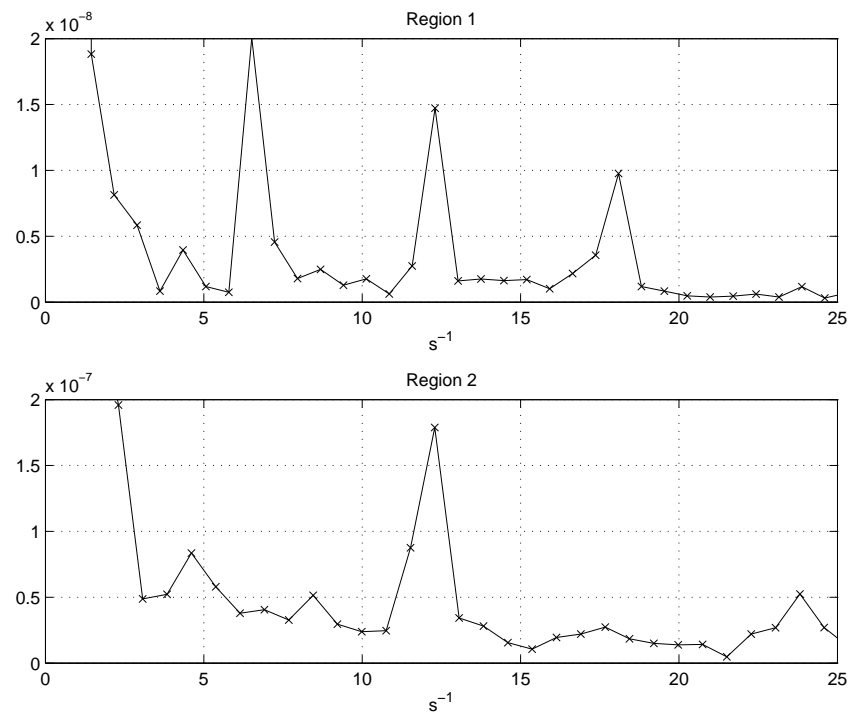

Fig. 5. The Fourier spectrum of the grid 1 currents, from Fig. 4, region 2 below $(81.0$ to $82.4 \mathrm{~km})$ and region 2 within the part of the dust layer where positive currents are measured $(82.7$ to $84.0 \mathrm{~km})$.

Below the dust layer the power at a frequency equal to the rotation frequency $\omega_{R}$ of the rocket is of the same magnitude as the power at $2 \omega_{R}$. The power at $3 \omega_{R}$ is also considerable while the $4 \omega_{R}$ contribution is negligible. Inside the dust layer, where large modulations correlated with the payload rotation are present, the power spectrum is dominated by the $2 \omega_{R}$ rotation. The $4 \omega_{R}$ is weak, but present, while the $\omega_{R}$ and $3 \omega_{R}$ frequencies are absent. If the current to G1 is interpreted solely as due to the charges of the impacting particles, we will show that the current modulation on the crossed wires of G1 must be four times the rocket spin. The magnitude of the modulation will also be very small, and far from what is observed. We find that the large current modulation can probably only be a result of secondary currents due to primary dust particle fragmentation, coupled with the effects of the geometry of the rotating and crossed grids when the coning is large. The front grid G1 of the dust probe consists (as does G2) of crossed grid wires of diameter $0.8 \mathrm{~mm}$ with a distance from wire centre to wire centre of $6.5 \mathrm{~mm}$. The pure geometrical coverage of the grids, at zero coning, compared to the total cross section of the probe, is $\sigma=0.23$. The grid wires in one parallel direction are all placed over another set of grid wires at $90^{\circ}$ to the first set of wires, so that there is an under and an over set of wires (see Fig. A1). This geometry is vital in our model, as it leads to a large fraction of the negatively charged fragments from the upper wires to be reabsorbed by the lower wires when the coning is high. In this way the secondary effects on the upper wires are suppressed, and the $2 \omega_{R}$ frequency becomes dominant, as observed.

In our calculations of the currents measured by G1 we will use a model which is similar to that indicated by the observations of Tomsic (2003), in order to account for secondary

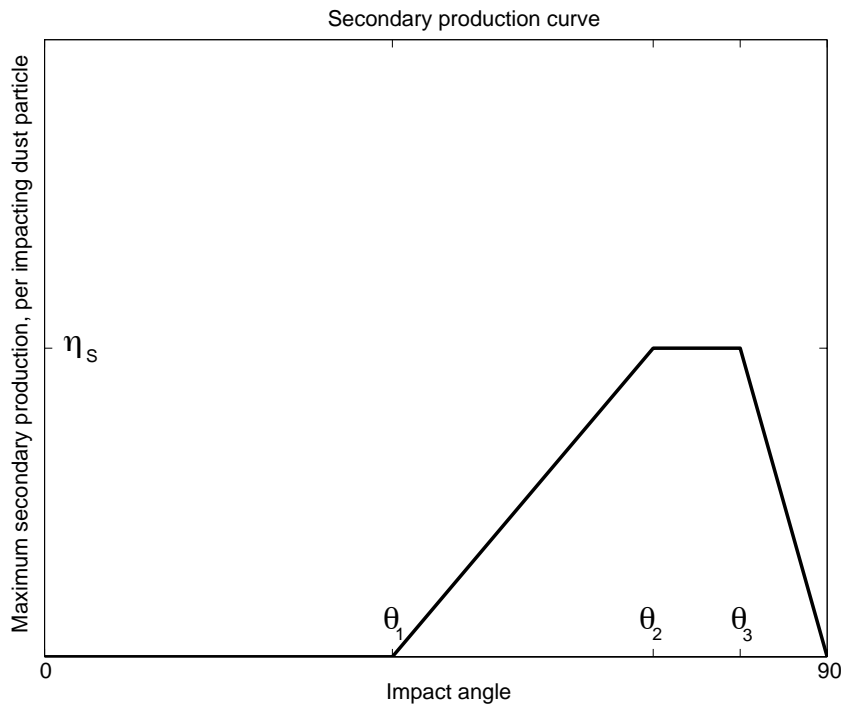

Fig. 6. The impact angle dependence for charged fragment production. The production of secondary fragments starts at impact angle $\theta_{1}$, peaks in the interval between $\theta_{2}$ and $\theta_{3}$ and goes to zero at $90^{\circ}$ impact angle.

charge production due to dust fragmentation. The relative secondary charge production $\eta_{S}$ is assumed to vary with the impact angle, as shown in Fig. 6. The values of $\eta_{S}$ are given relative to one incoming dust particle, so, for example, for a value $\eta_{S}=10$, the number of fragments carrying away a unit charge is 10 . The secondary production starts at an impact angle $\theta_{1}$, and increases with higher impact angles to a maximum between $\theta_{2}$ and $\theta_{3}$, and falls off to zero at impact angle $90^{\circ}$.

For the ice particles used by Tomsic (2003), $\theta_{1}$ is around $45^{\circ}$ while $\theta_{2}$ and $\theta_{3}$ are in the range $70-80^{\circ}$. The reason for this production curve is probably that the ice particles will totally sublimate at low impact angles, so that no fragments survive to carry away the secondary charge. Additionally, the ice particles apparently have to be larger than about 6 to $7 \mathrm{~nm}$ or they will sublimate fully also at larger impact angles. If mesospheric dust particles fragment, and each fragment contains one or more small particles of meteoritic origin, we find it likely that even if the water ice partly sublimates, the meteoric particles do not. We will therefore in our modelling allow for smaller values of $\theta_{1}$ than Tomsic (2003), and we will also assume that all small dust particles, or fragments of them, will produce surviving small meteoritic nuclei, or Hunten particles. This may also lead to a much larger production of secondary charges than for pure ice particles.

In the Appendix we develop a detailed model for the formation of the current $I_{\mathrm{G} 1}$ to $\mathrm{G} 1$. We integrate the impacts on the grid over all permissible impact angles, together with their secondary production. We also include the possibility that fragments from the top set of the parallel wires in G1 can be re-absorbed by the grid's lower set of parallel wires. 


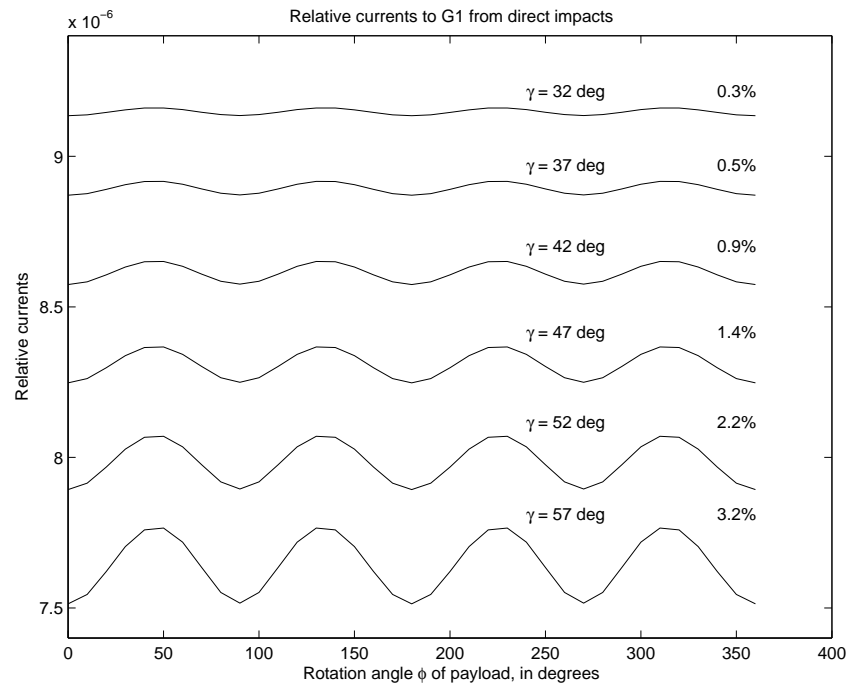

Fig. 7. Examples of how the currents to grid 1 would appear for a constant flux of charged particles onto the grid, without any effects of secondary currents due to charged fragment production. We show the resulting currents for several coning angles of the payload. The frequency of the modulation is clearly 4 times the payload spin rate and the modulation is very small, of the order of $1 \%$ only.

In order to demonstrate that the direct current due to impacting dust particles cannot reproduce the observations, we first calculated the variation of the direct current $I_{\mathrm{G} 1}$ (direct) with the use of Eq. (A4) (see Appendix) for different coning angles $\gamma$, and show the results in Fig. 7. The rotation modulation for a coning angle such as that of ECT-07 is now only of the order of $1 \%$, calculated as $\left.100\left(I_{\mathrm{G} 1}(\max )-I_{\mathrm{G} 1}(\min )\right)\right) / I_{\mathrm{G} 1}(\max )$. The dominant frequency is $4 \omega_{R}$. This can also be shown analytically by calculating the variation in the total effective area for the two grids as a function of payload rotation angle $\phi(t)$. This gives, to the highest order in $\sin \gamma$, a variation of $-\sin ^{4} \gamma \cos (4 \phi(t)) / 32$, with an average value of somewhat less than 1, again showing that the variation will be of the order of $1 \%$ and at a frequency of $4 \omega_{R}$. This is clearly nothing like the observed variations in $I_{\mathrm{G} 1}$ of up to $50 \%$ at $2 \omega_{R}$. To reproduce the observations, the contributions to the current from one set of the grid wires has to be strongly suppressed so that the other grid wires normal to them dominate the current. This would lead to a $2 \omega_{R}$ dominance for the $I_{\mathrm{G} 1}$ variation, which is what we observed. This is what happens if we have a re-absorption of fragments from the upper $X$-wires. We now calculate some examples of $I_{\mathrm{G} 1}$ (see Eq. (A18) in Appendix) as a function of $\phi(t)$ for different values of the relevant parameters (see the Appendix) when secondary currents are included. In Fig. 8 we show the results for two different coning angles $\gamma=42^{\circ}$ and $47^{\circ}$, for two different values of $b=1.5$ and 2.5 and for two different secondary production curves $\left[\theta_{1}, \theta_{2}, \theta_{3}\right]$. The factor $b$ is a measure of the effective cross section (Eq. A16) of a grid wire for the capture

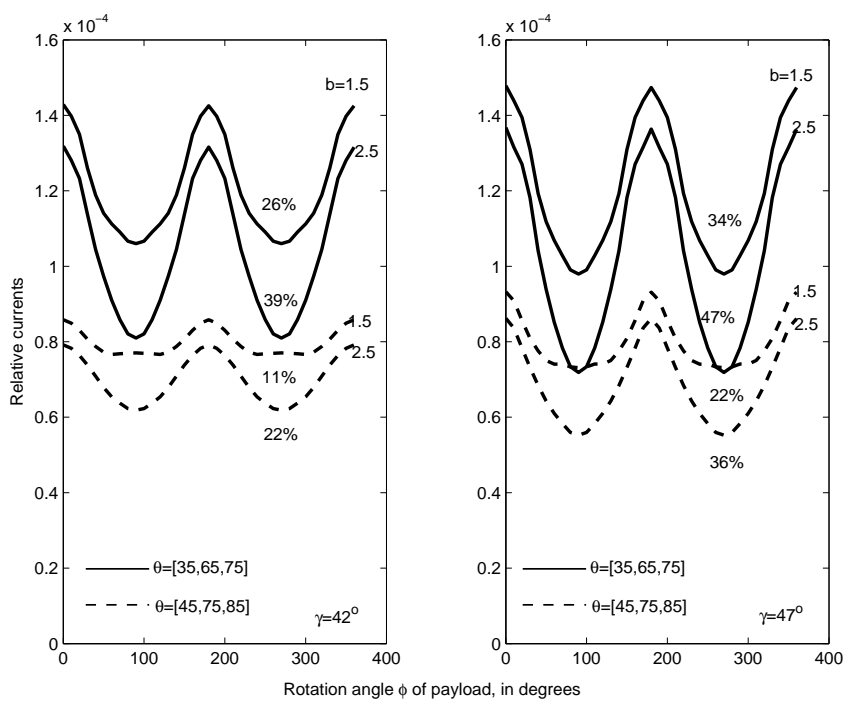

Fig. 8. Some examples where the secondary production and reabsorption of fragments are included. Only negatively charged fragments are produced, with a maximum secondary production $\eta_{S}=50$. The results are for two coning angles $\gamma$, for two values of the focusing parameter $b$ and two different secondary production curves $\left[\theta_{1} \theta_{2} \theta_{3}\right]$. The chosen parameters are given in the figures. The frequency of the modulation is now 2 times the payload spin rate with a considerable modulation.

of a negatively charged small fragment. A value of $b=1$ indicates that the fragments leaving the upper grid move in a straight line, while a value of $b>1$ is for cases where there is some attraction from the lower grid wires on the charged fragments from the upper grid. One secondary production curve of the form $[45,75,85]$ is chosen to illustrate the effect for a secondary production which may be representative for the impact of ice, while $[35,65,75]$ may be more relevant for the impact of meteoritic fragments on our grid wires. For all of these cases we use $\eta_{S}(\max )=50$. The direct impacts have a small influence on the total current. We also give the variation of the current in \% and note that it varies strongly with the secondary production curve, the focusing effect $b$, and the coning angle $\gamma$. We see that a modulation of $47 \%$ is achieved for a coning angle of $47^{\circ}$ with the use of a comparatively large focusing effect of $b=2.5$. A larger variation can be achieved if the secondary production is effective at even lower impact angles. These results demonstrate that even a moderate absorption of the secondary currents from the upper grid wires by the lower grid wires will suppress the $4 \omega_{R}$ modulation and make the $2 \omega_{R}$ modulation dominant, as observed. In Fig. 9 we have examined how the variation is dependent on the maximum secondary production $\eta_{S}$. We find that after an initial strong increase in the variation, as the secondary production starts to become dominant, there is only a small increase at $\eta_{S}>50$.

It is possible to produce large modulations of the observed current if we assume that only negatively charged secondary 


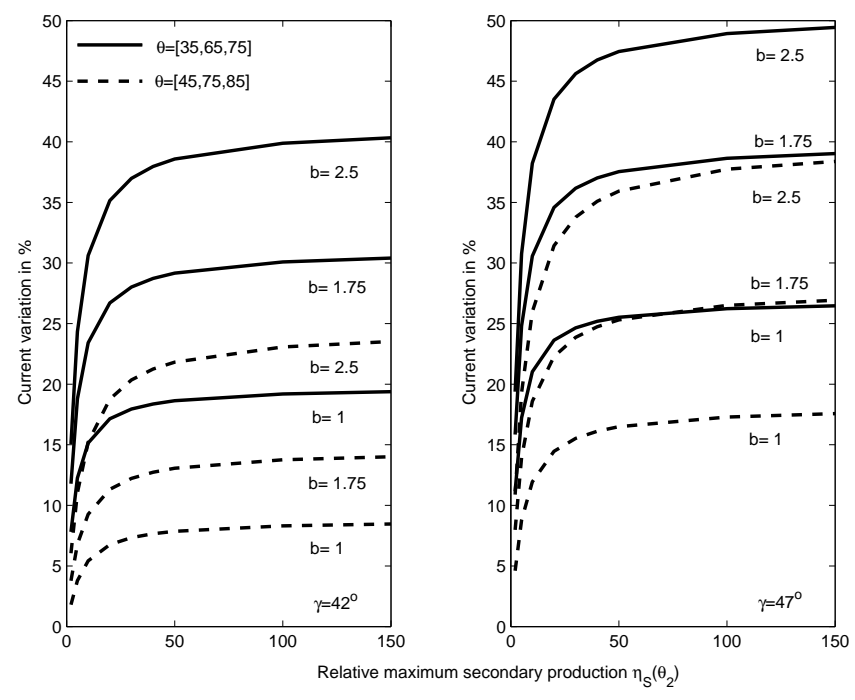

Fig. 9. The dependence of the current modulation on the maximum secondary production, for a different choice of coning angle, focusing on parameter and secondary production curves. The chosen parameters are given in the figures.

fragments are produced. This may not be the case, however, and the currents measured at the lower grid G2 indicate that positively charged secondary fragments are also produced at least in the lower parts of the dust layer. We see from Fig. 4 that this grid, which is at a negative potential of $-6.2 \mathrm{~V}$, measures predominantly positive currents in the lower half of the layer. Since grid 1 closes the interior to the ambient positive ions, and grid 2 is nearly completely shadowed by the probe walls due to the large coning, so that direct impacts will be limited, the natural explanation is that some positively charged fragments are also produced when dust particles hit G1 and fragment (e.g. Gridin et al., 2004). The positively charged fragments from G1 will be deflected by the G1 wires, and most will be ejected into the space between G1 and G2, where they will hit the probe walls or be attracted to G2. The positively charged fragments must be produced in considerably smaller numbers than the negative ones, in order to explain the observed positive currents to G1. Production of a smaller number of positively charged fragments will make it easier to reproduce the comparatively large current modulations, since they will contribute a negative current to G1 and thereby lower the average value of the current to this grid.

Although we realize that our assumed dust model and its effects at impacts on grid 1 of ECT-07 is quite speculative, we are confident that we have demonstrated that the observed currents from grid 1 cannot have been produced primarily by direct impacts of charged dust particles. A full model should include factors like a distribution of fragment sizes, a more realistic secondary production curve and a focusing factor $b$ which varies with impact conditions. In addition, a distribution in sizes of directly impacting particles should be included. However, the model as we have presented it should contain the major effects of a secondary production, and as such, serve as a test as to whether the observed variations of $I_{\mathrm{G} 1}$ can be explained by secondary production or not. In Fig. 10 we show an example where we have taken the first three peaks of the currents to G1, starting at about $82.45 \mathrm{~km}$ and ending at $82.7 \mathrm{~km}$ altitude, and roughly fitted the model results. The choice of production curve is driven by our need to reproduce the observations. Although the results do not vary dramatically for moderate changes in the production curve, we find that we always require a shift in the production curve towards smaller impact angles compared to the findings of Tomsic (2003). It appears that a moderate focusing of the fragments $(b>1)$ is also required. However, this depends on the value of the coning of the payload. We have chosen two models, the first having the production curve shape with $[35,55,75]$ for $\left[\theta_{1}, \theta_{2}, \theta_{3}\right]$ and coning angle $\gamma=42^{\circ}$. In the other example, we have used $[30,55,75]$ and increased the coning angle by $10^{\circ}$ relative to the quoted observed value. This was done because this increases the width of the modelled peaks, which seems to be required by the observations. Given the uncertainties in angle measurements by magnetometers on rocket payloads, we do not find it unlikely that the real coning could have been larger than the $42^{\circ}$, as given for ECT-07. The large modulation we observe is easily reproduced for $\gamma=42^{\circ}$, if we include both negative and positive fragments, but the current peaks become narrow. Without positive fragments it is difficult to produce a sufficiently large current modulation. The positive fragments must have a focusing parameter $b<1$ because they are repelled by the wires of G1. The value of the various parameters are given in the figure caption. In Fig. 11 we have fitted model results to observations in the upper part of the layer, between 83.38 and $83.63 \mathrm{~km}$. We have, as for Fig. 10, calculated model results for the two coning angles, $\gamma=42$ and $52^{\circ}$. We see that the required variation in this case can now be reproduced even if only negatively charged fragments are produced at impact on G1. The reason for this is that the current, due to directly impacting dust particles, is assumed to have a charge number $Z_{d}=-1$, and now has a larger effect in lowering the level of the positive current. However, the profiles appear to be too narrow, also in the case when $\gamma=52^{\circ}$. We therefore tried a secondary production curve of the form $[20,80,85]$. The results for such cases, also without the production of positive fragments, are fitted to the observations in Fig. 12. The width of the current peaks in the model results now agrees well with the observed current peaks, but it still appears that a coning angle larger than $42^{\circ}$ is required by the observations.

Our conclusions on the charging processes and formation of the currents to grid 1, for the payload ECT-07 with its high coning angle, can be summarized as:

1. In the dust layer from around the 82.2 to $83.7 \mathrm{~km}$ altitude, which consists of the entire dust layer, except for 


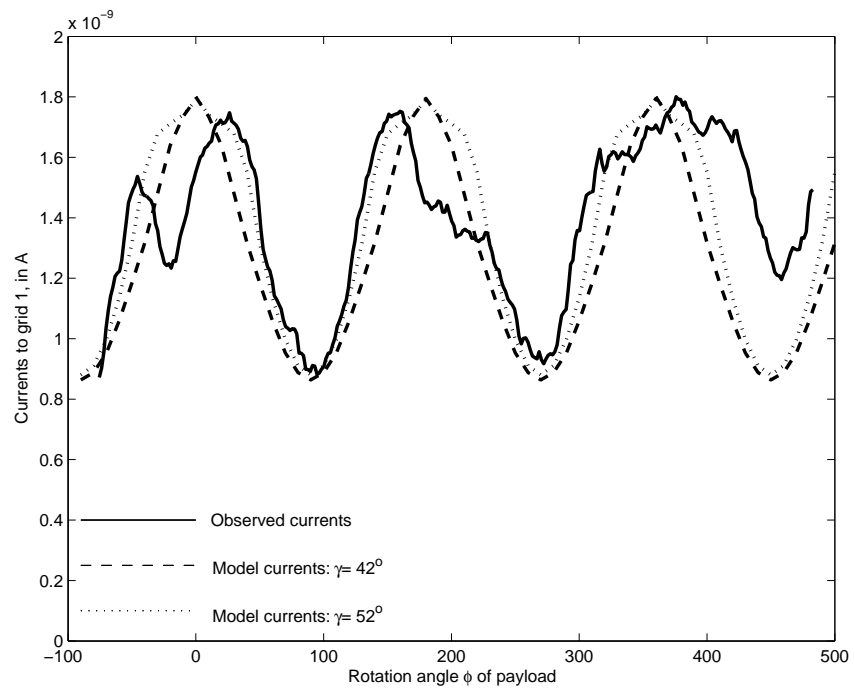

Fig. 10. Model calculations fitted to the measured currents measured by grid 1 in the lower part of the dust cloud between heights 82.45 to $82.7 \mathrm{~km}$. The model for the coning $\gamma=42^{\circ}$ uses the following parameters: maximum secondary production for negatively and positively charged fragments are $\eta_{S_{-}}=100$ and $\eta_{S_{+}}=25$, respectively, with corresponding focusing parameters $b_{-}=2.0$ and $b_{+}=0.5$. The secondary production follows $[35,55,75]$. For the higher coning at $\gamma=52^{\circ}$ the corresponding values are $\eta_{S-}=50$, $\eta_{S_{+}}=10, b_{-}=1.4, b_{+}=0.8$ and secondary production $[30,55,75]$.

the top, where grid 1 registers a negative current, the secondary charge production must dominate the current to grid 1 , and the strong modulation of the current to G1 at $2 \omega_{R}$ (see Fig. 4 ) is caused by the payload rotation.

2. The dependence of the secondary production on the impact angle must be shifted to smaller angles for impacts of PMSE dust particles compared to those which are valid for water-ice clusters (Tomsic, 2003) in order to explain the observed modulations.

3. In the lower part of the dust layer, we observed a modulation of the observed current to G1, together with positive currents on G2. This apparently requires that both negatively and positively charged fragments are produced when incoming dust particles impact G1 and therefore fragment. The production of negatively charged fragments must be dominant. In the upper part of the dust cloud the results can be modelled both with and without having to introduce the production of positively charged fragments.

4. It seems to be required that the motion of the fragmented charged dust particle fragments produced at grid 1 is affected by the electric field of that same grid. This causes the re-absorption of negatively charged fragments to be larger than if they were large enough to move in straight lines, while positively charged fragments will be re-

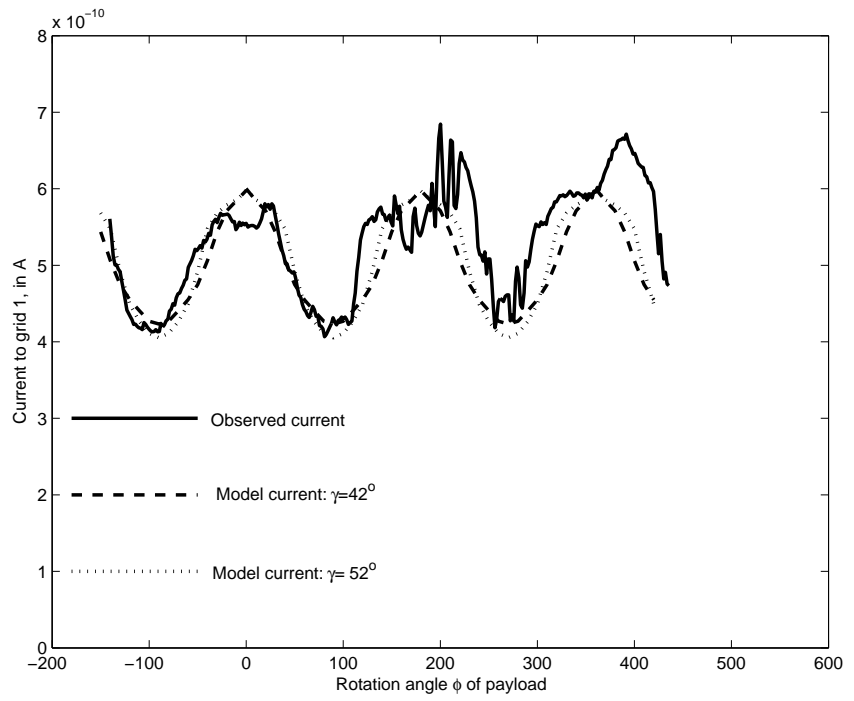

Fig. 11. Model calculations fitted to currents measured by grid 1 in the upper part of the dust cloud between heights 83.38 and $83.63 \mathrm{~km}$. As for Fig. 10 we have made models for two coning angles. We have for these cases not included any production of positively charged fragments; see discussion in text. For $\gamma=42^{\circ}$ the model parameters are $\eta_{S}=100, b=1.2$ and the secondary production curve $[30,55,75]$. For $\gamma=52^{\circ}$ we used $\eta_{S}=80, b=1.0$ and a secondary production curve $[30,70,80]$.

pelled by the wires and have a smaller re-absorption. For the charged fragments to be affected by the electric field of grid 1 wires, their sizes should be of the order of $1 \mathrm{~nm}$ or less if their charge number $Z_{s}$ is -1 . If we use the "best fit" results from Figs. 10 and 11 with $b=1.4$, and insert in Eq. (A17), using $V_{\mathrm{G} 1}=6.2$ Volt, we find $r_{s}=0.7 \mathrm{~nm}$ if $\rho_{s}=1000 \mathrm{~kg} \mathrm{~m}^{-3}$, and $0.5 \mathrm{~nm}$ if $\rho_{s}=3000 \mathrm{~kg} \mathrm{~m}^{-3}$. Since we expect that most of the water ice sublimates during the impact, these radii could correspond to the nucleus of meteoritic material, possibly so-called Hunten particles. We will later discuss the total fragment size $r_{f}$, consisting of one or more Hunten particles plus the water ice around them.

\section{Primary dust densities and sizes required for model results to fit observed currents to grid 1}

Based on our model results, we can now calculate the required density of primary dust particles impacting the rocket. Figure 8 shows, for the demonstration cases, the magnitude of the effective area which is expected for production of a net positive current to grid 1 when secondary production dominates. This figure shows (see Eqs. A6 and A16) that the relative current $I$ (secondary) $/ N_{d} V_{R} e$ can be regarded as an effective area for secondary charge production. The results are for one $X$ - and one $Y$-wire of one square (there is only one $X$ side and one $Y$ side contributing from each 


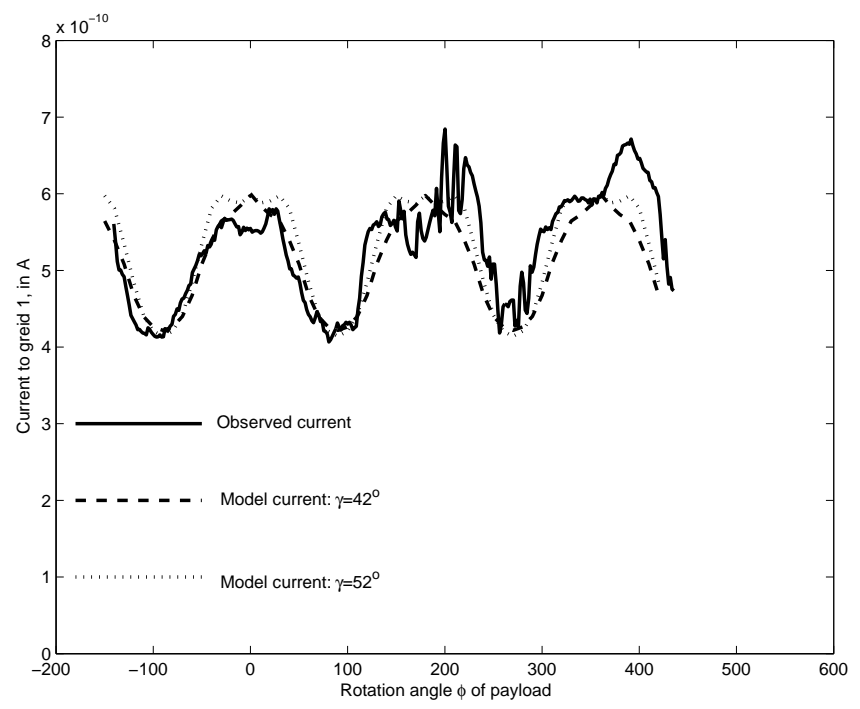

Fig. 12. Models for the same height interval as in Fig. 11, but in this case the secondary production curve has been chosen as [20, 80, 85] for both coning angles to investigate the consequence of a more extreme production. There is no production of positively charged fragments. The other parameters are for $\gamma=42^{\circ}$, chosen as $\eta_{S}=60$ and $b=1.4$ and for $\gamma=52^{\circ}, \eta_{S}=60$ and $b=1$.

square) when impacts are weighted with the production and re-absorption of secondary particles. This means that the impacting particles produce positive charge flux on these wires equal to the influx of dust particles multiplied with the effective area and the unit charge. For these demonstration cases the effective area is of the order of $10^{-4} \mathrm{~m}^{2}$. For the models, which are adapted to the observed currents to G1 in the lower parts of the dust layers, the maximum effective area during a cycle is $\operatorname{Ar}\left(42^{\circ}\right)=2.3 \times 10^{-4} \mathrm{~m}^{2}$, and $\operatorname{Ar}\left(52^{\circ}\right)=1.4 \times 10^{-4} \mathrm{~m}^{2}$ for the coning angles $\gamma=42^{\circ}$ and $52^{\circ}$, respectively. This also demonstrates that secondary production totally dominates the net current in our models. If no secondary particles were produced, as for the cases shown in Fig. 7, the net effective area would be of the order of $8 \times 10^{-6} \mathrm{~m}^{2}$. We can now find the primary dust densities which are required by our models to produce the observed current to G1 at the low end and at the high end of the dust layer. The observed maximum current to G1 in the lower part of the dust layer is $I_{\mathrm{G} 1}=1.8 \times 10^{-9} \mathrm{~A}$, as shown in Fig. 10. Since there are 206 squares in grid 1 and the influx of primary dust particles per $\mathrm{m}^{2}$ and per second is $N_{d} V_{R}$, we can find $N_{d}$ by solving $206 \mathrm{Ar} N_{d} V_{R} e=1.8 \times 10^{-9}$. We obtain for the first case with $\gamma=42^{\circ}$ that $N_{d}=2.6 \times 10^{8} \mathrm{~m}^{-3}$, and $N_{d}=4.3 \times 10^{8} \mathrm{~m}^{-3}$ for the second case with $\gamma=52^{\circ}$. The model calculations to reproduce the currents to G1 in the upper parts of the dust layer, with a maximum current of $I_{\mathrm{G} 1} \approx 6.5 \times 10^{-10} \mathrm{~A}$, require dust densities varying from $N_{d}=6 \times 10^{7} \mathrm{~m}^{-3}$ to $1.3 \times 10^{8} \mathrm{~m}^{-3}$. These dust number densities agree well with values found by von Cossart et al.
(1999), based on 3-color lidar observations of NLC. They quote number densities at various occasions when the NLC was observable by lidar (as it was during the launch ECT07 ), ranging from $N_{d}=2.3 \times 10^{7} \mathrm{~m}^{-3}$ to $1.1 \times 10^{9} \mathrm{~m}^{-3}$, with an average density of $\left\langle N_{d}\right\rangle=(8.2 \pm 5.2) \times 10^{7} \mathrm{~m}^{-3}$ and radius $\left\langle r_{d}\right\rangle \sim 50 \mathrm{~nm}$. If we assume that the dust particles fragment during impact into sub-particles of radius $r_{f}$, then the maximum number of negatively charged fragments is $\left(r_{d} / r_{f}\right)^{3} f$, where $f$ is the fraction of fragments carrying away a negative charge, or the probability that one fragment will be charged. If the icy fragments each contain one meteoritic Hunten particle (Hunten et al., 1980), we must also require that the number density of the Hunten particles locked in the dust particles is not much larger than a reasonable value for the maximum density $N_{H U}$ which has been estimated for the Hunten particles. Since it is possible that Hunten particles may have been swept up from a volume which is considerably larger than the volume of the dust cloud, we require that $N_{d}\left(r_{d} / r_{f}\right)^{3} \leq \varepsilon N_{H U}$. For the "sweeping up" factor if we use values from $\varepsilon=1$ to 10 , and a maximum density $N_{H U} \sim 10^{10} \mathrm{~m}^{-3}$, we obtain that the fragment sizes must be in the range from 5 to $10 \mathrm{~nm}$ when using the average values for $N_{d}$ and $r_{d}$ (von Cossart et al., 1999). This again requires a fractional ionization $f \geq \eta_{S}\left(r_{f} / r_{d}\right)^{3} \approx .05$ to 0.8 when we use $\eta_{S}$ from 50 to 100 . This means that for the lowest fragment charging probability, 1 out of 20 fragments must be charged, while at the highest probability, practically all fragments must be charged. Although we could obtain $f \sim 0.01$ by choosing larger values for $N_{H U}$ or $\varepsilon$, this still shows that our model requires at least 1 order (and possibly up to between 3 to 4 orders) of magnitude more efficient secondary charge production than for the impact of pure ice particles of similar sizes for the fragments.

However, there are obvious differences between the impact of our postulated dust particles and that of nonfragmenting pure water-ice clusters. The fragmentation process itself may lead to some charging of the fragments and the impact surface (e.g. Avila et al., 2005). Also, it is likely that if the nuclei of sub-particles are meteoritic of the origin, they will not sublimate during the impact, even though most or all of the condensed water ice on them may do so. The surviving number of fragments, or the nuclei of the sub-particles, should therefore be significantly higher for the model sub-particles than for water ice clusters of the same size and impact in similar numbers. For our postulated surviving fragments, consisting of meteoritic material, which may contain metals and silicates as well as other elements, there are, to our knowledge, no relevant impact ionization experiments with similar small particles and low velocities. We have, therefore, no experimental support for the required high fractional charging values other than the observations by the DUSTY probe on ECT-02 and ECT-07. 


\section{Discussion}

We are at present unable to ascertain if the model for the impacting dust particles, which resulted from our analysis, has any similarities to real mesospheric dust particles, or if other, possibly more realistic, dust models can also lead to the large secondary production which we find that our observations required. However, we would like to propose the following scenario to create a larger dust particle, consisting mainly of water ice, within which there are a considerable number of small Hunten particles of radius $r_{H} \lesssim 1 \mathrm{~nm}$ embedded. Our impact model, inspired by the requirements of the observations by ECT-02 and ECT-07, apparently implies that the large dust particles fragment during impact and that the fragments contain one or more Hunten particles. It is assumed that the ice, which is also the main part of the fragments, sublimates for most of the impacts, but that the Hunten particles survive and that a fraction of them carry away charge from the impact surfaces. In this model for the icy dust particles, which collect Hunten particles at a certain rate as they grow, the growth of the dust particle is almost exclusively due to the condensation of water vapour. The scenario which may produce the dust particles we proposed for ECT-07 is that ice particles start to form high up in the PMSE layer by water vapour condensing on sufficiently large Hunten particles. As these particles, on which water has condensed, fall downwards, they continue to grow by condensation of water vapour, but at the same time they also collide with uncharged small Hunten particles (which may be too small for effective condensation of water) (Rapp and Thomas, 2006) that attach to them, so that they become more or less evenly distributed throughout their volumes and on their surfaces, with an average interdistance of $\sim 2 r_{f}$, where $r_{f}$ is, as before, the average fragment size. For Hunten particles of radius $r_{H}$ to be accreted at a non-negligible rate onto the larger dust particles of radius $r_{d}$, they must either be neutral or of opposite charge. We neglect the possibility of them being positively charged, although this would lead to more effective capture, and therefore, the rate at which the neutral Hunten particles will collide with, and presumably stick to, a larger particle is

$\frac{d N_{H C}}{d t}=\pi r_{d}^{2} \beta N_{H} v_{H} \mathrm{~s}^{-1}$.

We have assumed that a fraction $\beta$ of the Hunten particles are neutral. The larger dust particles increase their radius mainly by the condensation of water vapour of mass, number density and thermal velocity, $n_{V}, m_{V}$ and $v_{V}$, respectively, at the rate

$$
\frac{d r_{d}}{d t}=\frac{n_{V} m_{V} v_{V}}{4 \rho_{d}}
$$

At the same time the accretion of neutral Hunten particles must proceed at a rate which keeps the embedded density of them at a value of $2 r_{f}^{-3} \mathrm{~m}^{-3}$, so that $d N_{H C} / d t \sim\left(4 \pi r_{d}^{2}\left(d r_{d} / d t\right)\right) /\left(2 r_{f}^{3}\right)$. From Eqs. (7) and (8) we find that to achieve this, the density $N_{H}$ of Hunten particles must be of the order of

$N_{H} \sim \frac{1}{8 \beta} \frac{n_{V} m_{V} v_{V}}{\rho_{d} r_{f}^{3} v_{H}}$

Using $\rho_{d}=1000 \mathrm{~kg} / \mathrm{m}^{3}, \quad n_{V}=5 \mathrm{ppmv} \sim 1.5 \times 10^{14} \mathrm{~m}^{-3}$, $v_{V}=400 \mathrm{~m} / \mathrm{s}, v_{H}=30 \mathrm{~m} / \mathrm{s}$ (for $r_{H} \sim 1 \mathrm{~nm}$ ) and $r_{f}=8 \mathrm{~nm}$, we obtain $N_{H} \sim 1.4 \times 10^{10} / \beta \mathrm{m}^{-3}$. For a high density of small Hunten particles, coexisting with larger dust particles, the fraction of them that are uncharged during normal PMSE conditions can easily be above 0.5 . This indicates that the dust model which seems to be indicated by the observations may be produceable in the mesosphere when the density of Hunten particles is of the order of a few times $10^{10} \mathrm{~m}^{-3}$ and the amount of water vapour present leads to a growth rate of the dust particle of a few nanometer per hour. If the growth rate is higher than this the density, $N_{H}$ must also be larger, in order to give the same proportion of Hunten particles in the dust particle. If the original small Hunten particles are also covered by a condensed ice-layer, this will lower the accretion rate and therefore also require a higher density $N_{H}$, in order to yield the same embedded density. Recent calculations on the ablation and condensation of meteoric material confirm that densities of $N_{H U}$ comparable to our requirements may well be present (Megner et al., 2006). Considering the capture of the smallest Hunten particles with a radius of just a fraction of a nanometer, they find that densities of $N_{H U}(0.2 \mathrm{~nm}) \sim 10^{11} \mathrm{~m}^{-3}$ may be present. If such small particles can carry away charge during impact, this may put less severe constraints on our model. They will more easily be captured and their density within the dust particle will be increased, and thus the requirement on the fractional ionization will be lower.

While our results do not exclude that mesospheric dust particles can be positively charged, we feel we have demonstrated that secondary production of charges must always be considered when dust particles impact rocket payloads or probes with a construction so that dust particles hit them at high impact angles. In some cases, when the dust particles are comparatively large, this may dominate the measured currents on such payloads. This clearly happened on the front grid G1 of ECT-07 and the neglect of this effect led to the initial wrong conclusion (Havnes et al., 1996) that the total currents to G1 were carried by impacting dust particles with a considerable positive charge. We cannot be absolutely certain which charge sign the primary dust particles had, but their charge numbers must have been low and most probably negative. We are also confident that the apparent increase in electron density within the dust layer, as measured by the CONE instrument (Fig. 2), originally taken as a sign of a photoelectron production process occurring there, charging the dust positive and thereby releasing electrons, was a result of a positive charging of the payload itself. Dust particles impacting the payload, with its high coning, will also fragment. 
The negative charges carried away by the surviving parts of the fragments would lead to an increase in the payload potential. With a fragment size of $\sim 1 \mathrm{~nm}$, their kinetic energy will be $\sim 10 \mathrm{eV}$, but if we allow for a distribution of fragment sizes, we may have energies several times this value. This would lift the CONE potential, which is at $+6 \mathrm{~V}$ with respect to the payload. An increase in this potential with respect to the ambient plasma will lead to an increased electron flux to the probe, which is what we observe.

\section{Conclusion}

We feel we have shown that secondary production of charge during impact of mesospheric dust particles on rocket probes and rocket payloads is an effect which can lead to misinterpretation of the ambient plasma and dust conditions, if not properly evaluated and taken into account. Our modelling, including secondary charge production, is capable of explaining measurements by the dust probe DUSTY (Havnes et al., 1996), where positive currents are detected by one of its grids, which cannot be explained by the direct impact of dust particles with no secondary production. A major potential difficulty with our results is that we require, for our mesospheric dust particles, a secondary charge production efficiency which is much larger than what is found in laboratory experiments for similarly sized pure water ice particles. On the other hand, the requirement by the model on reabsorption of secondary charges by the grid itself apparently indicates that the mesospheric dust particles, although most likely composed almost entirely by water ice, must still be very different from the pure ice particles in experiments. Our modelling has used a water ice particle interspersed with small meteoritic smoke (Hunten) particles, which fragments during impact into many smaller subparticles, each containing one or several smoke particles. The reason for a fragmentation could be the effect of the embedded Hunten particles on the structural strength of the icy dust particle structure and not necessarily that Hunten particles with an ice mantle are accreted and loosely stick to the icy dust particles. This would be a too slow process to lead to sufficiently rapid growth. While pure water ice particles below size 6-7 nm, which would be close to our inferred subparticle size, will totally sublimate, and larger particles will partly sublimate, the smoke particles embedded in the mesospheric dust particles should survive and may be capable of carrying away secondary charge.

We will also stress that our results allow for the possibility that we can find information on the relative dust sizes throughout the dust layer by measuring the secondary current and comparing it with the direct current from the incoming dust. If the secondary charge production due to the impact of mesospheric dust increases with dust size, as it does for water-ice particles in experiments, the relation between the secondary and direct current and its variation with height, should lead to information on the relative dust sizes throughout the layer. For the flight ECT-02 the parameters of Eq. (4), shown in Fig. 2, give a qualitative description of the relative sizes in the layer. With the exception of the measurements below $\sim 83.5 \mathrm{~km}$ and above $\sim 88 \mathrm{~km}$, where the denominator in $R_{S}$ is close to zero and where the dust layers end, a larger negative value of the ratio indicates larger dust particles, while positive values around 0.3 indicate small particles. We see that the largest particles are below the strong bite-out and also in its upper part and above it. The dust particle size decreases towards the top and bottom of the dust layer.

Although the DUSTY probe design provides enough information to separate the secondary and direct current, future DUSTY probes will be designed with more care to separate the two currents with better accuracy.

\section{Appendix A}

We examine the revolving grid wires, as shown in Fig. A1, and relate them to a fixed coordinate system $(x, y, z)$, where $z$ is along the payload axis and pointing towards the front of the payload. The rotating system $(X, Y, Z)$ of the payload also has the $\mathrm{Z}$-axis along the rocket axis while the $\mathrm{X}$ - and $\mathrm{Y}$ axes are directed along the wires of the upper and lower layer of the grid wires of G1, respectively. This system rotates at the spin rate of the rocket. The incoming dust has a velocity direction in the $y z$-plane with an angle to the Z- and z-axes equal to the payload coning angle, which for ECT-07 was $\gamma \approx 42^{\circ}$. The impact angle $\theta_{i x}$ for the dust on the $\mathrm{X}$-axis grid is found from

$\cos \theta_{i x}=\cos \xi \cos \gamma-\sin \xi \sin \gamma \cos \phi(t)$

and for the Y-grid

$\cos \theta_{i y}=\cos \xi \cos \gamma+\sin \xi \sin \gamma \sin \phi(t)$.

The angle $\phi(t)$ is the angle between the rotating and fixed coordinate systems. The angles $\theta_{i x}$ and $\theta_{i y}$ are now the respective impact angles, i.e. the angles between the velocity direction of the incoming dust particle and the grid wire normal, at the chosen impact position, given by the angle $\xi$ between this normal and the z-axis. For a part of a grid wire, equal to the length $l_{W}=6.5 \mathrm{~mm}$ of one side in one of the squares which are formed by the crossed wires, of radius $R_{W}=0.4 \mathrm{~mm}$, the effective area of a strip along it, at position $\xi$ and with angular width $d \xi$, seen from the incoming dust, will be

$d A_{\text {eff }}(j)=l_{W} R_{W} \cos \theta_{i j} d \xi, \quad j=x, y$

The current contributed by the directly impacting dust on one upper wire of a square, plus that from one side of the square from the lower wire will be

$$
\begin{aligned}
I_{G 1}(\text { direct })= & I_{0} \int d A_{\mathrm{eff}}(x) \\
& +I_{0} \int(1-\Delta) d A_{\mathrm{eff}}(y) .
\end{aligned}
$$


We integrate around the wires, but exclude values where $\cos \theta_{x}$ or $\cos \theta_{y}$ are negative, since this corresponds to parts of the wire which are in the shadow with respect to the incoming dust particles. There is also a small fraction $\Delta$ of the lower wire which is shadowed by the upper wire. This will vary from a value of $\Delta=2 R_{W} / l_{W} \cos \gamma$ for $\phi(t)=0$ or $\pi$, to $2 R_{W} / l_{W}$ for $\phi(t)=\pi / 2$ or $3 \pi / 2$. We will assume that the variation is sinusoidal. The current due to incoming charged dust onto a unit area moving with the rocket velocity $V_{R}$ with its normal parallel to the rocket velocity direction is

$I_{0}=N_{d} Z_{d} e V_{R}$.

Here $N_{d}$ and $Z_{d}$ are the dust density and dust charge number, respectively, while $e$ is the unit charge and $V_{R}=900 \mathrm{~m} / \mathrm{s}$ is the rocket velocity.

We now consider the current due to the fragmentation of impacting dust, where the relative secondary production is a function of impact angle $\eta_{S}\left(\theta_{i}\right)$. We have

$$
\begin{array}{r}
I \text { (secondary) }=N_{d} V_{R} e \int \eta_{S}\left(\theta_{i x}\right) d A_{\mathrm{eff}}(x) \\
+N_{d} V_{R} e \int(1-\Delta) \eta_{S}\left(\theta_{i y}\right) d A_{\mathrm{eff}}(y),
\end{array}
$$

if a possible re-absorption of some of the fragments is neglected. We also note that the ratio $I$ (secondary) $/ N_{d} V_{R} e$ can be regarded as an effective area of one $X$ plus $Y$ wire in one square of the grid for production of secondary charges. For ECT-07 with its large coning, it is clear that many of the fragments produced by impacts on the upper $X$-wires, where they leave with a velocity approximately in the plane tangential to the impact point, will hit the lower wires and be re-absorbed. They will therefore not contribute to the current given by Eq. (A6). It is this absorption which we find leads to the observed current to G1 being dominated by a $2 \omega_{R}$ frequency with a large amplitude, and not by a $4 \omega_{R}$ frequency of low amplitude, as follows from Eq. (A4) when only directly impacting charged dust contribute to the measured current (see Fig. 7). There is also a small correction, since those fragments which leave along planes with a normal close to the rocket axis (Z-axis) may hit the neighbouring parallel grid and lead to self-absorption of secondary fragments. This has, however, little effect on the net current, mainly since impacts at these conditions, for the coning of ECT-07, normally have small impact angles which produce few secondary charged particles. The full length of an impact strip (see Eq. A3) on a $X$-wire at one side of a square is $l_{W}$. The length of this strip from which secondary particles will hit the lower $Y$-wires is, for a given rotation angle $\phi(t)$ and coning angle $\gamma$, dependent on the orientation of the tangential plane at the strip position $\xi$ and the angle $\alpha$ the velocity of the outgoing fragments makes with the strip. If we first assume that all dust particles, incoming and fragments, move in straight lines, the angle $\alpha$ can readily be found as follows: the direction of incoming dust is

$\hat{\boldsymbol{v}}_{\text {in }}=-\sin \gamma \hat{\boldsymbol{y}}-\cos \gamma \hat{\boldsymbol{z}}$

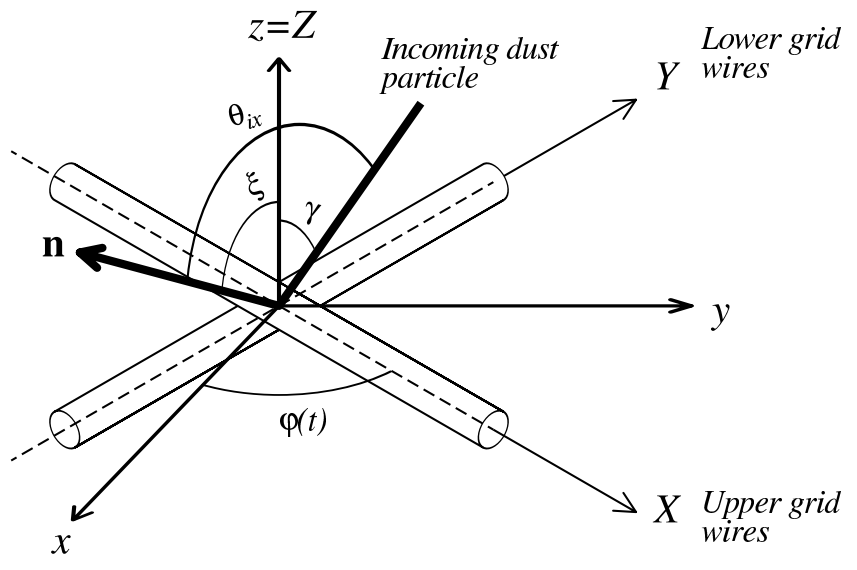

Fig. A1. The coordinate system used to describe the coordinate system of the rotating grid 1 wires and the geometry of impacts. For details see text.

and the normal to an impact point at angle $\xi$ is

$$
\begin{gathered}
\hat{\boldsymbol{n}}=\quad \sin \xi \sin \phi(t) \hat{\boldsymbol{x}} \\
-\sin \xi \cos \phi(t) \hat{\boldsymbol{y}} \\
+\cos \xi \hat{z} .
\end{gathered}
$$

The direction of the velocity of the fragments, if we assume that they leave at a tangent to the grid wire at the impact point, is given by

$\boldsymbol{v}_{\text {out }}=\hat{\boldsymbol{n}} \times\left(\hat{\boldsymbol{v}}_{\text {in }} \times \hat{\boldsymbol{n}}\right)$.

The fragments come from a strip of length $L$ and width $R_{W} d \xi$ on an upper grid wire. A unit vector along this strip is given by

$\hat{\boldsymbol{L}}=\cos \phi \hat{\boldsymbol{x}}+\sin \phi \hat{\boldsymbol{y}}$

and the angle $\alpha$ between this strip and the velocity of the fragment is found from

$\cos \alpha=-\frac{\hat{\boldsymbol{L}} \cdot \boldsymbol{v}_{\text {out }}}{\left|\boldsymbol{v}_{\text {out }}\right|}$.

The plane in which the fragments move (tangential at the impact point) will cut the lower grid wire in an ellipse given by

$l_{x}^{2}+l_{y}^{2} \sin ^{2} \xi=R_{W}^{2}$.

Here the $l_{x}$ coordinates are in the tangential plane in the direction of the $X$-wires, while the $l_{y}$ coordinates are normal to this. For an angle $\alpha$ the points on each side of the ellipse, where fragments hit with a grazing incidence, are found by $d l_{y} / d l_{x}=\tan \alpha$. These two points are found to be, in the coordinate system of Eq. (A12),

$l_{y t}= \pm \frac{R_{W} \cot \alpha}{\sin \xi\left(\sin ^{2} \xi+\cot ^{2} \alpha\right)^{1 / 2}}$ 
$l_{x t}= \pm \frac{R_{W} \sin \alpha}{\sin \xi\left(\sin ^{2} \xi+\cot ^{2} \alpha\right)^{1 / 2}}$

The grazing points map back along the tangents to the strip on the upper wire to give a length

$$
\begin{aligned}
L & =2\left|l_{y t}\right| \tan \alpha+2\left|l_{x t}\right| \\
& =\frac{2 R_{W}}{\sin \xi}\left(\sin ^{2} \xi+\cot ^{2} \alpha\right)^{1 / 2} .
\end{aligned}
$$

Charged fragments coming from this length of the strip which we consider will be re-absorbed and not contribute to the net current to G1. If the strip length we find by Eq. (A15) is longer than the square side length $l_{W}=6.5 \mathrm{~mm}$, we put $L=l_{W}$. In the integration for the secondary current of Eq. (A6) we now multiply the integrand of the first integral by the factor $\left(1-L / l_{W}\right)$, so that the parts of the upper grid, which deliver secondary fragments that are re-absorbed by the lower grid wires, do not contribute to the current to G1.

Before computing the relative currents for different cases, we also argue that if the fragments have low enough masses, the negatively charged fragments from the upper grids can be affected by the lower grid potential, so that there will be some focusing of particles. In this discussion we have neglected the possible effect of the air flow around the probe on the fragments. Such a flow will deflect small incoming dust particles in front of the probe, but the problem may be much less severe for the hypothetical small fragments since they are produced slightly inside the probe. We also expect that the influence of air flow will be small for a fragment moving from the dust impact point on the upper grid wire to the closest lower grid wire, a distance of $\lesssim 6-10 \mathrm{~mm}$ only. For a full description of small dust and fragment orbits one will have to consider the influence of the airflow outside and inside the probe (Horányi et al., 1999). This problem should be addressed in future work if our hypothesis of fragmentation and small particle production is correct. With a focusing of fragments, the value of $L$ from Eq. (A15) will be increased. As a rough measure of the focusing effect we use the standard formula for the effective relative cross section radius $b$, compared to that of a sphere with a surface potential $V_{\mathrm{G} 1}$, for a collision with a charged fragment particle of charge $Z_{s} e$, mass $m_{s}=\frac{4 \pi}{3} \rho_{s} r_{s}^{3}$ and velocity $v_{s}$ (Spitzer, 1978; Whipple, 1981)

$b=\left(1-\frac{2 Z_{s} e V_{G 1}}{m_{s} v_{s}^{2}}\right)^{1 / 2}$.

This means that for a significant focusing effect to be present, we require that the kinetic energy is comparable to, or less than, the charge of the fragment multiplied with the effective potential of the grid wires.

We use $Z_{s}=-1$, and for the velocity of the fragments we use a fixed value of $v_{s}=700 \mathrm{~m} / \mathrm{s}$, which corresponds to the velocity component of the incoming dust of velocity equal to the rocket velocity $900 \mathrm{~m} / \mathrm{s}$, along the tangent at impact if the impact angle is $\sim 50^{\circ}$

$$
\left(\frac{V_{G 1}}{10 \text { Volt }}\right)\left(\frac{\rho_{s}}{1000 \mathrm{~kg} \mathrm{~m}^{-3}}\right)^{-1} r_{s}^{-3}(\mathrm{~nm}) \geq 0.6,
$$

showing that the sizes of the fragments should be around a nanometer or smaller if focusing is to be of importance. Changing the fragment velocity to 800 or $600 \mathrm{~m} / \mathrm{s}$ produces a change in the requirement on the fragment sizes of $\pm 10 \%$. The radius $r_{s}$ in Eq. (A17) may not be equal to that of a subparticle, but more like that of its nucleus (Hunten particle), if the water-ice condensate on it sublimates during the impact. The focusing effect will increase the length of the absorption strip $L$ given in Eq. (A15) by the factor $b$ of Eq. (A16), since its effect is to increase the effective radius of the grid wire. We still have the condition that if $b L>l_{W}$, we put $b L=l_{W}$. The expression for calculating the current from one $X$-wire and one $Y$-wire in one of the squares of the crossed grid wires now becomes

$$
\begin{aligned}
I_{\mathrm{G} 1}=I_{\mathrm{G} 1}(\text { direct }) & \\
+N_{d} V_{R} e & {\left[\int\left(1-\frac{b L}{l_{W}}\right) \eta_{S}\left(\theta_{i x}\right) d A_{\mathrm{eff}}(x)\right.} \\
& \left.+\int(1-\Delta) \eta_{S}\left(\theta_{i y}\right) d A_{\mathrm{eff}}(y)\right] .
\end{aligned}
$$

The fragment velocity $v_{s}$ is approximately equal to the component of the incoming dust particle velocity $V_{R}$, along the tangent at the impact point. The use of $v_{s}=0.75 V_{R}$ is for an impact angle $\eta \sim 50^{\circ}$. For higher impact angles, at $\eta \approx 90^{\circ}$, the right-hand side of Eq. (A16) becomes $\sim 1.1$.

Acknowledgements. We would like to thank J. Gumbel for critical and very useful comments on an early version of this paper, and the referees for valuable comments which helped improve it.

Topical Editor U.-P. Hoppe thanks two referees for their help in evaluating this paper.

\section{References}

Andersson, P. U. and Pettersson, J. B. C.: Ionization of water clusters by collisions with graphite surfaces, Zeitschrift für Physik D, 41, 57-62, doi:10.1007/s004600050289, 1997.

Avila, E. E., Saunders, C. P. R., Bax-Norman, H., and Castellano, N. E.: Charge sign reversal in irregular ice particle-graupel collisions, Geophys. Res. Lett., 32, L01801, 1-4, doi:10.1029/ 2004GL020761, 2005.

Blix, T. A.: Small Scale Plasma and Charged Aerosol Variations and Their Importance for Polar Mesosphere Summer Echoes, Adv. Space Res., 24, 537-546, 1999.

Blix, T. A., Thrane, E. V., and Andreassen, O.: In situ measurements of the fine-scale structure and turbulence in the mesosphere and lower thermosphere by means of electrostatic positive ion probes, J. Geophys. Res., 95, 5533-5548, 1990. 
Brasseur, G. and Solomon, S.: Aeronomy of the middle atmosphere: Chemistry and physics of the stratosphere and mesosphere, vol. 3, Dordrecht, D. Reidel Publishing Co., 1986, 457 p., 1986.

Cho, J. Y. N. and Röttger, J.: An updated review of polar mesosphere summer echoes: Observation, theory, and their relationship to noctilucent clouds and subvisible aerosols, J. Geophys. Res., 102, 2001-2020, doi:10.1029/96JD02030, 1997.

Dalmann, B.-K., Grün, E., Kissel, J., and Dietzel, H.: The ioncomposition of the plasma produced by impacts of fast dust particles, Plan. Space Sci., 25, 135-147, 1977.

Gadsden, M. and Schröder, W.: Noctilucent Clouds, Physics and chemistry in space 18, Springer-Verlag, 1989.

Gridin, V. V., Gebhardt, C. R., Tomsic, A., Schechter, I., Schröder, H., and Kompa, K. L.: Surface impact induced fragmentation and charging of neat and mixed clusters of $\mathrm{SO} 2$ and $\mathrm{H} 2 \mathrm{O}$, Int. J. Mass Spectrom., 232, 1-7, 2004.

Gumbel, J. and Witt, G.: In situ measurements of the vertical structure of a noctilucent cloud, Geophys. Res. Lett., 25, 493-496, 1998.

Havnes, O., Trøim, J., Blix, T., Mortensen, W., Næsheim, L. I., Thrane, E., and Tønnesen, T.: First detection of charged dust particles in the Earth's mesosphere, J. Geophys. Res., 101, 10839 10 847, doi:10.1029/96JA00003, 1996.

Horányi, M., Gumbel, J., Witt, G., and Robertson, S.: Simulation of rocket-borne particle measurements in the mesosphere, Geophys. Res. Lett., 26, 1537-1540, doi:10.1029/1999GL900298, 1999.

Hunten, D. M., Turco, R. P., and Toon, O. B.: Smoke and dust particles of meteoric origin in the mesosphere and stratosphere, J. Atmos. Sci., 37, 1342-1357, 1980.

Megner, L., Rapp, M., and Gumbel, J.: Distribution of meteoric smoke - sensitivity to microphysical properties and atmospheric conditions, Atmos. Chem. Phys., 6, 4415-4426, 2006, http://www.atmos-chem-phys.net/6/4415/2006/.

Mitchell, J. D., Croskey, C. L., and Goldberg, R. A.: Evidence for charged aerosols and associated meter-scale structure in identified PMSE/NLC regions, Geophys. Res. Lett., 28, 1423-1426, doi:10.1029/2000GL012685, 2001.
Pedersen, A., Trøim, J., and Kane, J.: Rocket measurement showing removal of electrons above the mesopause in summer at high latitudes, Planet. Space Sci., 18, 945-947, 1969.

Rapp, M. and Lübken, F.-J.: Polar mesosphere summer echoes (PMSE): review of observations and current understanding, Atmos. Chem. Phys., 4, 2601-2633, 2004, http://www.atmos-chem-phys.net/4/2601/2004/.

Rapp, M. and Thomas, G. E.: Modeling the microphysics of mesospheric ice particles: Assessment of current capabilities and basic sensitivities, J. Atmos. Terr. Phys., 68, 715-744, doi:10.1016/j.jastp.2005.10.015, 2006.

Smiley, B., Rapp, M., Blix, T. A., Robertson, S., Horanyi, M., Latteck, R., and Fiedler, J.: Charge and size distribution of mesospheric aerosol particles measured inside NLC and PMSE during MIDAS MaCWAVE 2002, J. Atmos. Solar-Terr. Phys., 68(1), 114-123, 2006.

Spitzer, L. J.: Physical Processes in the Interstellar Medium, John Wiley, 1978.

Tomsic, A.: Collisions between water clusters and surfaces, Dr. filos. thesis, Gothenburg University, Gothenburg, 2003.

Ulwick, J. C., Baker, K. D., Kelley, M. C., Balsley, B. B., and Ecklund, W. L.: Comparison of simultaneous MST radar and electron density probe measurements during STATE, J. Geophys. Res., 93, 6989-7000, 1988.

von Cossart, G., Fiedler, J., and von Zahn, U.: Size distributions of NLC particles as determined from 3-color observations of NLC by ground-based lidar, Geophys. Res. Lett., 26, 1513-1516, 1999.

Vostrikov, A. A., Zadorozhny, A. M., Dubov, D. Y., Witt, G., Kazakova, I. V., Bragin, O. A., Kazakov, V. G., Kikhtenko, V. N., and Tyutin, A. A.: Ionization of water clusters by collision with surface, Z. Phys. D, 40(1-4), 542-545, 1997.

Whipple, E. C.: Potentials of surfaces in space, Reports of Progress in Physics, 44, 1197-1250, 1981.

Zadorozhny, A. M., Tyutin, A. A., Witt, G., Wilhelm, N., Walchli, U., Cho, J. Y. N., and Swartz, W. E.: Electric field measurements in the vicinity of noctilucent clouds and PMSE, Geophys. Res. Lett., 20, 2299-2302, 1993. 